\title{
Characteristics of gettering process in multicrystalline Si wafers with combined porous $\mathrm{Si} / \mathrm{Al}$ getters
}

\author{
A. Sarikov, V. Naseka \\ V. Lashkaryov Institute of Semiconductor Physics, NAS of Ukraine \\ 45, prospect Nauky, 03028 Kyiv, Ukraine \\ Phonelfax:+38 (044)525-62-90; e-mail:sarikov@isp.kiev.ua; victor_naseka@meta.ua
}

\begin{abstract}
In this work, the gettering process in the multicrystalline Si wafers by the combined getter structures of the porous $\mathrm{Si}$ and $\mathrm{Al}$ layers during annealings at temperatures 600 up to $750{ }^{\circ} \mathrm{C}$ has been theoretically studied. A kinetic model based on the diffusion equation has been developed, and the characteristics of increase in the minority charge carrier diffusion length as a result of gettering annealings have been determined. The obtained results are useful for technology of multicrystalline Si solar cells to improve their properties.
\end{abstract}

Keywords: gettering, multicrystalline Si, diffusion problem, combined getter.

Manuscript received 15.11.11; revised manuscript received 20.12.11; accepted for publication 26.01.12; published online 29.02.12.

\section{Introduction}

In crystalline $\mathrm{Si}$ solar cell technology, a great attention is given to the control over the recombination active metal impurities that cause degradation of the lifetime inherent to minority charge carriers in Si wafers. These impurities are present in initial $\mathrm{Si}$ material as well as can be additionally incorporated during technological wafer processing [1]. Among especially deteriorating metal impurities, there are transient metals, namely: iron, chromium, gold as well as heavy metals such as vanadium, molybdenum, wolfram, and titanium, which affect the characteristics of p-type Si used for solar cell production already at the concentrations as low as $10^{12}-$ $10^{14} \mathrm{~cm}^{-3}[2,3]$.

To reduce the concentration of metal impurities in $\mathrm{Si}$ wafers, the gettering procedures are included in technological routes of solar cell production. Increase of the lifetime of minority charge carriers is required over the entire Si wafer thickness. Therefore, external getters are mostly used in solar cell technology, in particular, the Al layer getters deposited onto the back side of $\mathrm{Si}$ wafers [1]. The gettering effect is obtained due to the several orders of magnitude higher solubility of metals in Al than in crystalline $\mathrm{Si}$. A decrease in the metal concentration in Si takes place due to metal diffusion in the wafer and its capture by the getter during annealings at elevated temperatures.
Earlier, we have suggested the modified getter structure that consists of porous $\mathrm{Si}$ with $\mathrm{Al}$ layer deposited on its top (combined getter) [4,5]. For multicrystalline $\mathrm{Si}$ wafers, this structure has demonstrated better performance as for the improvement of the recombination properties of minority charge carriers (increase in their diffusion length and lifetime) comparing to the traditional Al getters. In this work, we theoretically analyze the kinetics of gettering by the combined getter structures in the multicrystalline $\mathrm{Si}$ wafers in the framework of diffusion model and determine the respective process characteristics.

\section{Experimental}

We used the p-type multicrystalline Si wafers with the resistivity of $10 \mathrm{Ohm} \cdot \mathrm{cm}$ and the average grain size of $10 \mu \mathrm{m}$. Bilayer getter structures were formed on the back side of wafers by using the stain chemical etching in the decorating solution $\mathrm{HF}: \mathrm{HNO}_{3}: \mathrm{H}_{2} \mathrm{O}=1: \mathrm{X}: 1$ (X ranging from 0.001 to $10 \%$ by volume) with subsequent $\mathrm{Al}$ deposition onto the etched porous $\mathrm{Si}$ surface by vacuum thermal evaporation. The thicknesses of porous $\mathrm{Si}$ and Al layers were within the range 0.5 up to $1 \mu \mathrm{m}$.

$\mathrm{Si}$ wafers with created getter structures were annealed in Ar atmosphere at the temperatures 600 up to $950{ }^{\circ} \mathrm{C}$ for $30 \mathrm{~min}$. The gettering effect was controlled by measuring the diffusion length of minority charge 
carriers by using the capacitor photovoltage method [6] before and after annealings. Shown in Fig. 1 is the experimental temperature dependence of the relative diffusion length for minority charge carriers in the multicrystalline $\mathrm{Si}$ wafers. As can be seen from the figure, there are two branches of this dependence. The gettering effect is enchanced with increasing the annealing temperature up to about $750{ }^{\circ} \mathrm{C}$ (increase in the minority carrier diffusion length) and is weaker at higher temperatures due to saturation of getter and its decreasing capacity with respect to Si wafer $[1,7]$. We used the low temperature data of this dependence $\left(T \leq 750{ }^{\circ} \mathrm{C}\right)$ for the description in the framework of theoretical model and determination of the characteristics of gettering process in the multicrystalline $\mathrm{Si}$ wafers with the combined getter structures of porous $\mathrm{Si}$ and Al layers.

\section{Model}

In the framework of diffusion model, the kinetics of evolution of the concentration of recombination active metal impurity in Si wafer during gettering annealings is described by the equation of the second Fick law as follows:

$\frac{\partial C_{i m p}}{\partial t}=D_{i m p} \frac{\partial^{2} C_{i m p}}{\partial x^{2}}$.

Here, $C_{i m p}=C_{i m p}(x, t)$ is the concentration of recombination active metal impurity in the $\mathrm{Si}$ wafer and $D_{\text {imp }}$ is its diffusivity.

Solution of Eq. (1) requires additionally the boundary and the initial conditions. The boundary condition on the front (getter free) wafer surface $(x=0)$ is composed taking into account zero impurity flow through the wafer surface:

$\frac{\partial C_{i m p}(0, t)}{\partial x}=0$.

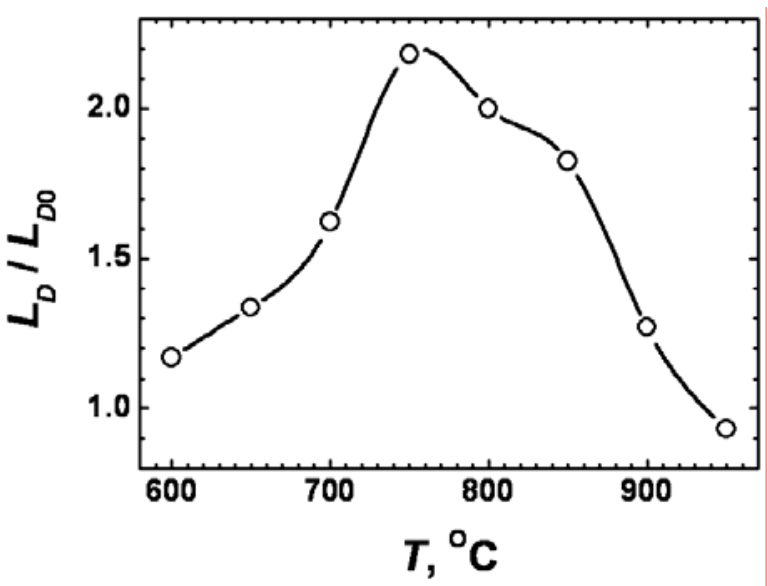

Fig. 1. Experimental temperature dependence of the relative diffusion length for minority charge carriers after 30-min gettering annealings of multicrystalline $\mathrm{Si}$ wafers with porous $\mathrm{Si} / \mathrm{Al}$ getter structures.

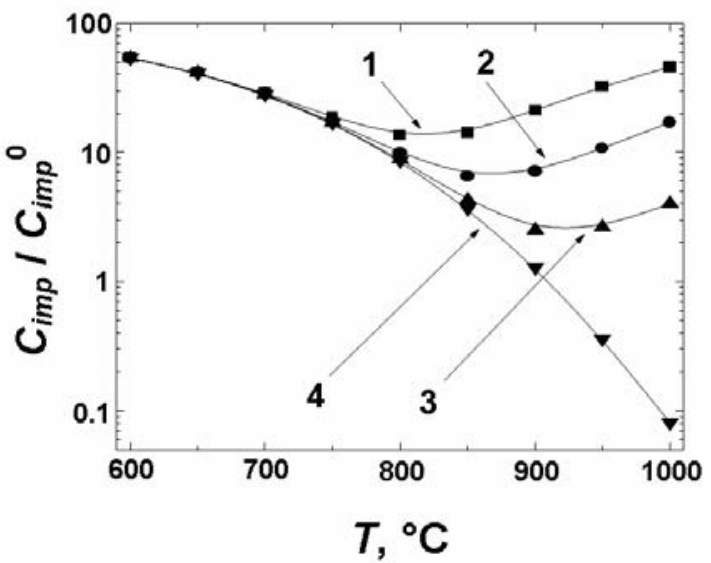

Fig. 2. Calculated temperature dependences of the effect of $\mathrm{Al}$ gettering in Si wafers for different getter thicknesses: $1-0.5$, $2-2,3-10 \mu \mathrm{m}$, and $4-$ infinitely thick getter. Annealing time is $30 \mathrm{~min}$. The $\mathrm{Si}$ wafer thickness is $300 \mu \mathrm{m}$.

The boundary condition on the back side of $\mathrm{Si}$ wafer ( $x=d_{\mathrm{Si}}, d_{\mathrm{Si}}$ being the $\mathrm{Si}$ wafer thickness) describes the kinetics of impurity capture by the getter. We have simplified the problem for the actual experimental conditions. Fig. 2 shows the calculated temperature dependences of the residual iron concentration in $\mathrm{Si}$ wafers annealed for $30 \mathrm{~min}$ at different $\mathrm{Al}$ getter thicknesses [7]. One can see that the gettering effects caused by the 0.5 to $3 \mu \mathrm{m}$ thick Al layers have almost no difference as compared to the effect from infinite getter in the temperature range 600 to $750{ }^{\circ} \mathrm{C}$. The gettering process for a given annealing time and mentioned (and higher) Al layer thicknesses within the temperature range 600 to $750{ }^{\circ} \mathrm{C}$ is controlled by the kinetics of impurity supply to the getter and not by the getter capacity with respect to Si wafer. Therefore, the getter can be considered infinite in our problem and the boundary condition on the back side of $\mathrm{Si}$ wafer acquires the following form:

$C_{\text {imp }}\left(d_{\mathrm{Si}}, t\right)=0$.

It should be noted that the approximation used here is valid without restrictions for thicker getters and shorter annealing times, whereas its validity for thinner getters and longer annealing times has to be verified.

The initial condition determines the impurity concentration in every point of Si wafer before gettering annealings. Assuming the homogeneous impurity distribution $C_{i m p}(x, 0)=C_{i m p}^{0}$.

The solution of diffusion problem (1)-(3) is significantly simplified using the normalized quantities:

$$
\frac{\partial v}{\partial \theta}=\frac{\partial^{2} v}{\partial \xi^{2}}
$$

where $v=v(\xi, \theta)=\frac{C_{i m p}}{C_{i m p}^{0}}$ is the normalized impurity 
concentration, $\xi=\frac{x}{d_{\mathrm{Si}}}$ coordinate, and $\theta=\frac{D_{i m p}}{d_{\mathrm{Si}}^{2}} t$ time, respectively. The boundary conditions (2) and (3) for the normalized problem acquire the following form:

$\frac{\partial v(0, \theta)}{\partial \theta}=0$

$v(1, \theta)=0$

and the initial condition for the system (4)-(6) is written as $v(\xi, 0)=1$.

To compare the calculation results with experimental data, the latter were transformed as follows. For the lifetime of minority charge carriers in $\mathrm{Si}$ wafers, the following relation is valid [8]:

$\frac{1}{\tau}=\frac{1}{\tau_{g}}+\frac{1}{\tau_{n g}}$

where $\tau, \tau_{g}$, and $\tau_{n g}$ are the determined minority charge carrier lifetime, the lifetimes in gettered and nongettered samples, respectively. The lifetime in the gettered ones results from heavy metal impurities that can be removed by gettering. The lifetime in the nongettered ones results from recombination of minority charge carriers with defects inside the Si wafer as well as on the front and back wafer sides. It cannot be improved by gettering.

The lifetime of minority charge carriers after gettering is inversely proportional to the heavy metal impurity concentration, capable of being removed (and, hence, to its normalized concentration), $C_{i m p} \sim v \sim \frac{1}{\tau_{g}}$.

From this

$C_{i m p}=$ const $\cdot\left(\frac{1}{\tau}-\frac{1}{\tau_{n g}}\right)$.

Since $\tau_{g}<\tau_{n g}$ in the multicrystalline $\mathrm{Si}$, $C_{i m p} \sim \mathcal{v} \sim \frac{1}{\tau}=\frac{D}{L_{D}^{2}}$, where $D$ is the diffusivity and $L_{D}$ is the diffusion length of minority charge carriers, respectively. So, the ratio of the concentrations of impurity atoms after and before gettering annealings of multicrystalline $\mathrm{Si}$ wafers can be considered as equal to the ratio of the squares of the diffusion lengths of minority charge carriers measured before and after gettering:

$\frac{C_{i m p}}{C_{i m p}^{0}}=\mathrm{v}=\frac{L_{D 0}^{2}}{L_{D}^{2}}$

with $L_{D 0}$ and $L_{D}$ being the diffusion lengths of minority charge carriers before and after gettering, respectively.

The results of calculations were compared to the experimental data in the following way. Eq. (4) with the boundary conditions (5) and (6) was solved numerically. The values of normalized impurity concentrations in all spatial mesh points as well as its total value for all the points were calculated at every time step. The ratio of the total normalized impurity concentration at a given time step to its value before gettering was compared to the experimental value of the ratio of the squares of minority charge carrier diffusion lengths before and after annealing at a given temperature. The calculation process was continued until the coincidence of these mentioned values. The normalized time of this event was fixed for every annealing temperature. Using the values of $\theta^{0}$ as well as the experimental annealing time $t^{0}=30 \mathrm{~min}$ and the Si wafer thickness $d_{\mathrm{Si}}=300 \mu \mathrm{m}$, the values of $D_{i m p}$ for each temperature were calculated using the formula:

$D_{\text {imp }}=\frac{d_{\mathrm{Si}}^{2}}{t^{0}} \theta^{0}$.

The obtained values of $D_{i m p}$ were fitted using the typical Arrhenius dependence on the temperature: $D_{i m p}=D_{i m p}^{0} \exp \left(-\frac{E_{d}}{k T}\right)$, and the values of $D_{i m p}^{0}$ and $E_{d}$, the pre-exponential term and the activation energy of diffusion process describing the kinetics of gettering in the multicrystalline $\mathrm{Si}$ wafers by the combined porous $\mathrm{Si} / \mathrm{Al}$ structures were determined from this fitting.

The advantage in formulation of the diffusion problem in the form (4)-(6) is the independence of solution on the impurity type, its initial concentration, and the annealing temperature. The independence on the impurity type is stipulated by the absence in Eq. (4) of the terms that explicitly contain certain impurity characteristics (such as, e.g., the impurity diffusivity and solubility). By analogy, the absence of terms with explicit temperature dependence makes the solution to be independent of changes in the annealing temperature, and only one run of calculations is required. The independence of the initial impurity concentration is stipulated by that we deal with only the ratios of concentrations before and after the gettering and not with their absolute values.

\section{Results and discussion}

Modelling results enabled to determine the parameters of the diffusion problem describing the gettering process in the multicrystalline Si wafers by the combined getters of porous $\mathrm{Si}$ and $\mathrm{Al}$ layers during annealings at the temperatures 600 to $750{ }^{\circ} \mathrm{C}$. Fig. 3 shows the temperature dependence of the values of diffusion coefficients in the problem calculated using the expression (10) as well as the result of approximation of these values by using the Arrhenius dependence. One may see that the calculated diffusivity values are well fitted by the straight line in the semilogarithmic coordinates that allows one to conclude that the gettering kinetics at the temperatures under consideration can be described by the diffusion process with the following 


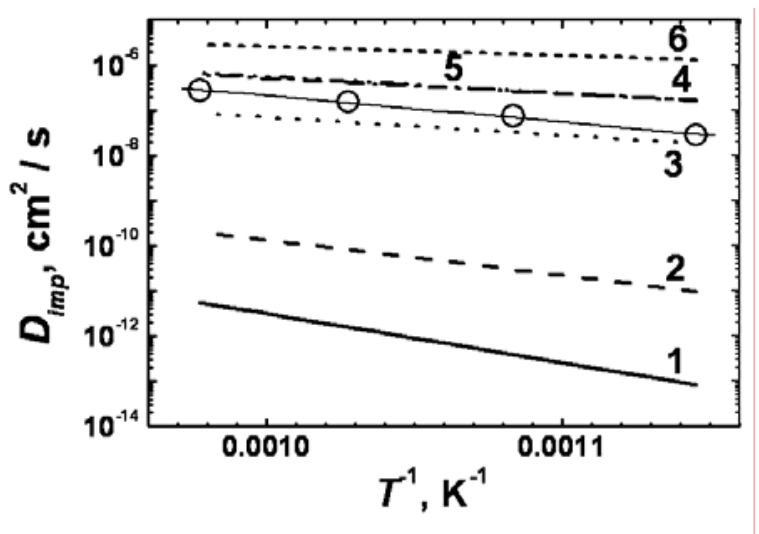

Fig. 3. Temperature dependences of diffusion coefficients of problem calculated by formula (10) (dots) and the dependences of the diffusion coefficients of main recombination active metal impurities in Si: 1 - titanium, 2 - vanadium, 3 chromium, 4 - iron, 5 - manganese, and 6 -gold.

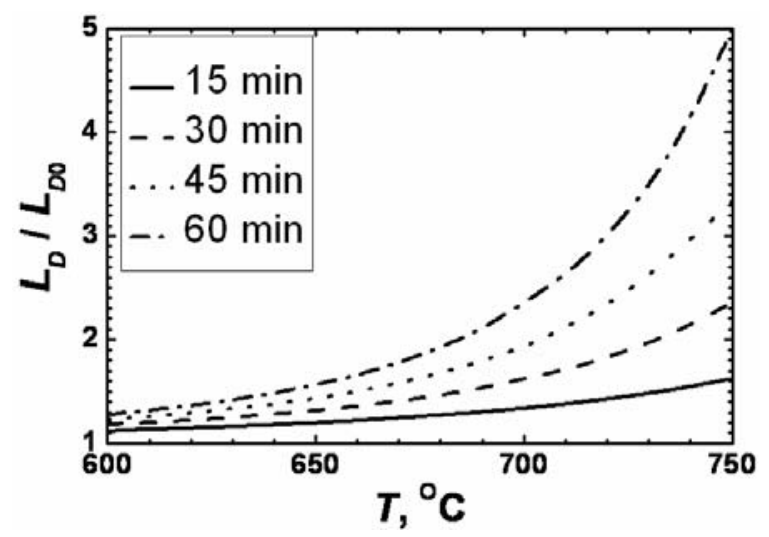

Fig. 4. Calculated temperature dependences of the relative diffusion length of minority charge carriers after various times of gettering annealings of multicrystalline $\mathrm{Si}$ wafers with porous $\mathrm{Si} / \mathrm{Al}$ getter structures.

characteristics: $D_{i m p}^{0}=0.278 \mathrm{~cm}^{2} / \mathrm{s}$ and $E_{d}=1.22 \mathrm{eV}$. Besides, Fig. 3 also presents the temperature dependences of the diffusivities of a number of most typical lifetimes for killing metal impurities in $\operatorname{Si}[9,10]$. As may be seen from this figure, the diffusivities of typical metals in $\mathrm{Si}$ do not correspond to $D_{\text {imp }}$. This means that the physical mechanism of gettering in the multicrystalline $\mathrm{Si}$ wafers with using the combined porous $\mathrm{Si} / \mathrm{Al}$ getters cannot be regarded as plain diffusion of the interstitial impurity of one type but corresponds to a more complex process.

Secondary ion mass spectrometry found out several main types of metal impurities in the multicrystalline $\mathrm{Si}$ wafers at the concentrations that lead to significant deterioration of their recombination properties [3]. These impurities are titanium, vanadium, chromium, iron, manganese, and gold. The concentrations of them also exceed their solubilities at the gettering temperatures that leads to the impurity precipitation and formation of complexes with other atoms and defects (such as, e.g., $\mathrm{Fe}-\mathrm{B}$ pairs). For the gettering effect, the metal atoms should be released from the complexes and precipitates in the interstitial state, in which they diffuse to the getter and are captured there. It is highly probable that in the multicrystalline $\mathrm{Si}$ wafers all the abovementioned impurities participate in the gettering process interacting with a number of capturing centres and precipitates. The modelling parameters determined in this work are therefore some effective parameters that do not characterize certain physical mechanism or impurity but the gettering process as a whole. They enable to predict the relative diffusion length of minority charge carriers for different thicknesses of porous $\mathrm{Si} / \mathrm{Al}$ getters and annealing times of multicrystalline $\mathrm{Si}$ wafers. As the example, Fig. 4 shows the calculated temperature dependences of the relative diffusion length of minority charge carriers for different times of gettering, if assuming the condition (3) is valid. The obtained results can be used in technology of multicrystalline Si solar cells with improved performances.

\section{Conclusion}

In this work, we have theoretically studied the kinetics of the gettering process in the multicrystalline $\mathrm{Si}$ wafers by the combined getter structures of porous $\mathrm{Si}$ with $\mathrm{Al}$ layers as a result of annealings at the temperatures 600 to $950{ }^{\circ} \mathrm{C}$. The gettering effect is enchanced for the temperatures up to about $750{ }^{\circ} \mathrm{C}$ when the process is governed by the impurity supply to the getter and not by the getter capacity. In this range, the process under consideration can be described using the diffusion model, which enables to determine its characteristics. The obtained results are important to improve recombination properties of multicrystalline $\mathrm{Si}$ solar cells.

\section{Acknowledgement}

This work has been carried out in the framework of the State Targeted Scientific and Technological Program "Creation of Chemical and Metallurgical Branch of the Production of Pure Silicon Material" during 2011 to 2015 (project 1-3.3 "Development and implementation of the technology for the formation of stress-free homogeneous Si ingots").

\section{References}

1. C. del Canizo and A. Luque, A comprehensive model for the gettering of lifetime-killing impurities in silicon // J. Electrochem. Soc. 147, p. 2685-2692 (2000).

2. K. Graff, Metal Impurities in Silicon-Device Fabrication. Springer-Verlag, Berlin, 1995.

\section{(C) 2012, V. Lashkaryov Institute of Semiconductor Physics, National Academy of Sciences of Ukraine}


3. V.G. Popov, Solar cells based on multicrystalline silicon // Semiconductor Physics, Quantum Electronics, and Optoelectronics 3, p. 479-488 (2000).

4. V.G. Litovchenko, N.I. Klyui, A.A. Evtukh, A.A. Efremov, A.V. Sarikov, V.G. Popov, V.P. Kostylyov, Yu.V. Rasamakin, Ch. Häßler, and W. Koch, Solar cells prepared on multicrystalline silicon subjected to new gettering and passivation treatments // Solar Energy Mater. and Solar Cells, 72, p. 343-351 (2002).

5. A. Efremov, A. Evtukh, N. Klyui, V. Litovchenko, V. Popov, Yu. Rassamakin, A. Sarikov, Ch. Häßler, and W. Koch, Effective gettering mechanisms in solar multicrystalline materials // Solid State Phenomena, 82-84, p. 719-726 (2002).

6. Standard test methods for minority carrier diffusion length in extrinsic semiconductors by measurement of steady state surface photovoltage, In: Annual Book of ASTM Standards, Section 10, Electrical
Insulation and Electronics, Vol. 10.05 Electronics (II), Designation: F 391-96 (1997).

7. A. Sarikov, Study of the formation mechanisms and physical properties of semiconductor structures with developed surface. Candidate of Physical and Mathematical Science thesis, Institute of Semiconductor Physics, NAS of Ukraine, Kyiv (2002).

8. A. Luque, A. Moehlecke, R. Lagos, and C. del Canizo, Segregation model for Si gettering by Al // Phys. Status Solidi (a), 155, p. $43-49$ (1996).

9. Diffusion in Silicon - 10 Years of Research, Ed. D.J. Fisher, Scitec Publications, Zuerich-Uetikon, Switzerland, 1998.

10. B.I. Boltaks, Diffusion and Point Defects in Semiconductors. Nauka, Leningrad, 1972 (in Russian).

11. A. Sarikov, V. Naseka, and V. Litovchenko, Influence of precipitates on the kinetics of iron gettering from the $\mathrm{Si}$ wafers by the Al layers // Phys. Status Solidi (c), 8, p. 767-770 (2011). 